\title{
Experimental analysis of magnetic field effects on compressor energy saving cooling system
}

\author{
Rakesh Kumar Sidheshware ${ }^{1, *}$, S Ganesan ${ }^{1}$, and Virendra K Bhojwani ${ }^{2}$ \\ ${ }^{1}$ Sathyabama Institute of Science and Technology, Chennai 600119, Tamil Nadu, India \\ ${ }^{2}$ MIT School of Engineering, MIT ADT University, Pune 412201, Maharashtra, India
}

\begin{abstract}
A research on different refrigerants with and without influence of the magnetic field is provided in this paper. The magnetic field degree viz is four gauss. The condenser exit row was $3000,6000,9000$ and 12000 gauss. Magnetic fields at condenser exit lines are found to reduce the consumption of the compressor energy. Through applying magnet fields on R134a, compressor energy savings was observed up to $1.35 \%, 6.23 \%, 5.40 \%$ and $4.19 \%$, respectively, in gauss levels of 3000,6000, 9000 and 12000, while R152a savings in energy levels were up $13.10 \%, 15.59 \%$ and $25.86 \%$ and R407a saving in power up to $7.3 \%, 9.19 \%, 13.05 \%$ and $12.40 \%$. The coolant R152a saves energy in contrast to other coolant compressors. As the magnetic field strength increases, a vapor refrigeration system decreases the energy consumption of the compressor.
\end{abstract}

\section{Intoduction}

Magnetic field effects on refrigerants are determined by its chemical composition and physical thermal properties such as viscosity, surface tension, thermal conductivity and heat. The highest measurable heat and thermal conductivity of coolants responds little and vice versa to the magnetic field. (Samuel M. Sami, R.J.Kita, 2005). The magnetic effect on the condensing outlet tube increases the cooling effect and reduces the compressor power. The compressor needs more power to remove liquid coolant from the compressor due to incomplete coolant evaporation. When the condenser has been magnetically applied, its cooling properties are modified and the evaporation frequency of liquid coolant is increased. The liquid coolant boiling in the compressor can therefore be reduced. (A Kotb, H.E.Saad2014).

The magnetic field effects are studied and its effect on vapor compression systems performance is analyzed in some modern refrigerant mixtures. (Samuel M. Sami, Shawn Aucoin, 2003). The impact of magnetic field on cooling mix R290 and R600a shows that magnetic field reduces energy cons-umption of the compressor and is dependent on strength of the magnetic field. The growing magnetic strength reduces compressor power and increases COP.

The effect of the magnetic field on the refrigerant mixes depends on the composition and boiling point of the mixture. (Kolandavel MANI, Velappan SELLADURAI,

\footnotetext{
*orresponding author: rakesh1987thermal@gmail.com
}

2008). Vapor compression depends on the efficiency of the coolant. For high COP, liquid thermal conductivity should be high, liquid and molecular weight should be low, and the latentheat vapor density. The vapor compression device is very prone to the normal hot spot, fluid-viscosity, thermal conductivity, density of vapour and critical temperature. (Rotchana Prapainop,K O Suen, 2012).

As the magnet strength increases, the effect of the magnetic field on hydrocarbon, viscosity and surrounding pressure decreases with increasing mass flow rate and thus heat transfer in the condenser and evaporator. (HejunGuo, Zhizhong Liu, Yunchao Chen, Rujie Yao).

The air conditioning unit output under the different strength of the magnetic field is having a positive effect on COP. (Mr. Amod P. Shrotri. Mr. Nikhil S. Mane, Mr. Vishal P. Patil, Mr. Sawan A. Wani, 2015).

\section{Apparatus and Procedure}

Figure1 demonstrates the experimental set up of a magnet-applied vapor compression system for liquid line. A hermetically screened compressor is used during the experimental setup. The condensing system will change the cooling stage from vapor to liquid once the condenser is installed. Then the use of a capillary tube eliminates high-pressure liquid coolant. The agitator is used for a uniform water temperature in the water tank. 


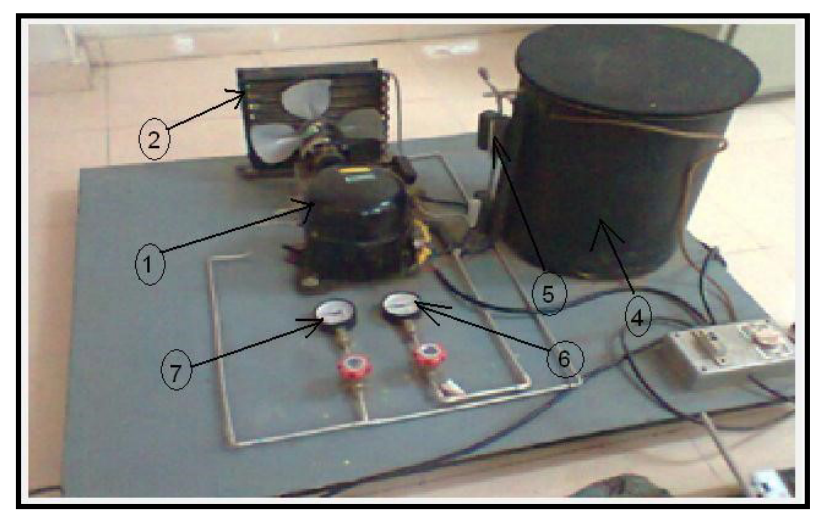

Fig.1. Setup of VCC system (1.compressor, 2.condenser 3.Capillary tube, 4.Evaporator, 5.Magnets, 6 .Suction pressure gauge, 7.Discharge pressure gauge)

In this setup four magnetic elements are used with each of 3000 Gauss level. The magnet is located at condenser exit line in increasing order of 3000, 6000, 9000 and 12000 gauss level. For mounting the magnet at exit condenser row, magnet separator is used. Under the same operating environment, different refrigerant types will be checked.

Water is held at $24 \mathrm{C}$ for processing and cooled down to $8 \mathrm{C}$ in the water tank. At different points of the device, temperature readings are reported at a period of 10 minutes. For various gauzes, the separate reading is taken. For each coolant, the same process is repeated.

\section{Results and discussion}

The output of the vapor compression process is used as baseline information without adding a magnetic field. On the exit line of the condenser is applied the first magnetic intensity at a rate of 3000 gauss and reading is registered.

To research the effect on compressor energy when the magnetic force is applied on the condenser exit line and the increased magnetic power order.

\subsection{Compressor Power for different refrigerants}

Due to the incomplete evaporation of coolants, the vapour compression system compressor absorbs additional power. If any liquid refrigerant exits the compressor, the compressor absorbs more liquid coolant with more energy to boil. If magnetic field is applied to the condenser exit line, its vaporization increases the amount of liquid cold and reduces the energy consumption of the compressor. It rising compressor power by increasing the gauss rate.

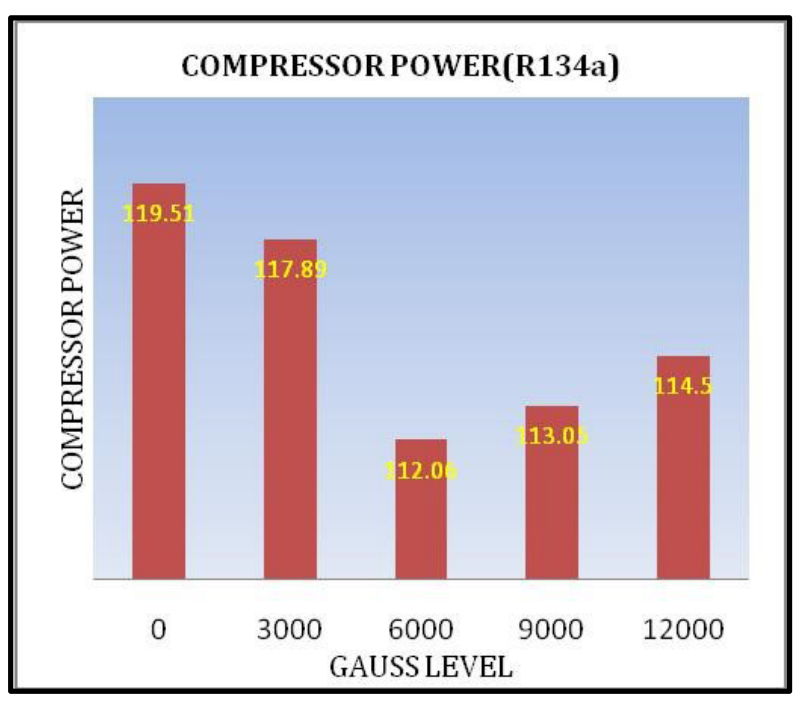

Fig. 2. Compressor Power (R134a)

Figure 2 shows the power of the compressor versus the magnetic field strength for the R134a. The average compressor efficiency for gauss rates 3000 is reduced by $1.35 \%$ relative to the non-magnet field. Average compressor energy falls by $6.23 \%, 5.40 \%$ respectively and 4.19 per cent for gauss rates 6000,9000 and 12000 .

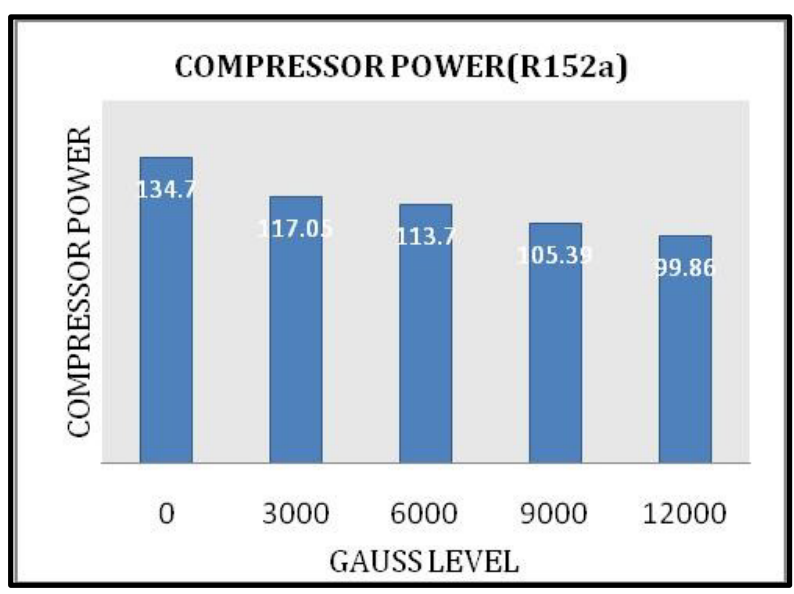

Fig. 3. Compressor Power (R152a)

The power vs. magnetic field strength for R152a is shown in Figure3. The rate of the gauss increases the compressor power. Average compressor power for gauss level 3000 decreased by 13.10 percent compared to the non-magnetic field. The average compressor power for gauss rates 6000,9000 and 12000 is $15.59 \%$, $21.75 \%$ and $25.86 \%$, respectively. 


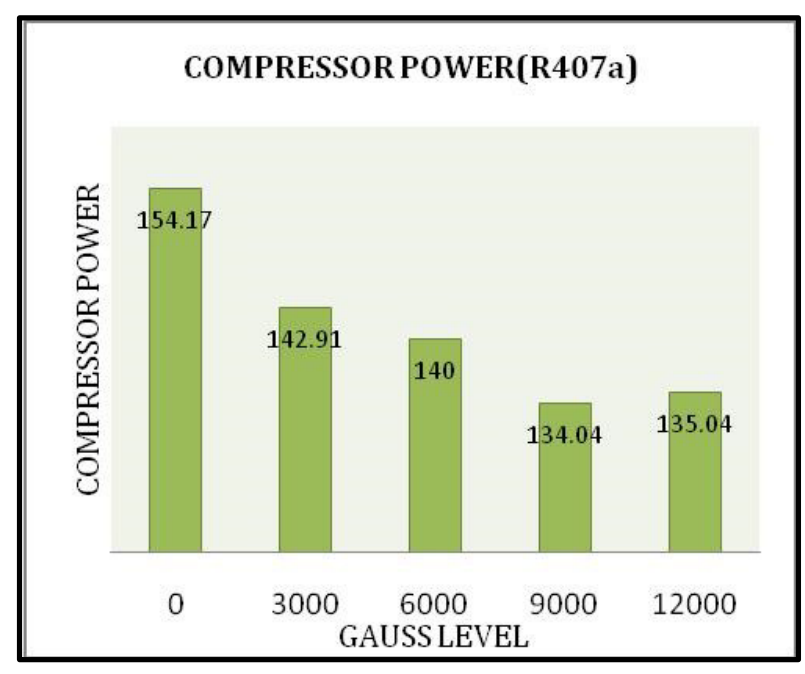

Fig. 4. Compressor Power (R407a)

The compressor power versus the magnetic field strength for the R407a is shown in figure 4. The average compressor strength of gauss level 3000 has been decreased by $7.3 \%$ compared to no magnet field. The average compressor capacity at Gauss rate 6000, 9000, 12000 and 13000 reduces respectively by $9.19 \%, 13.05 \%$ and $12.40 \%$.

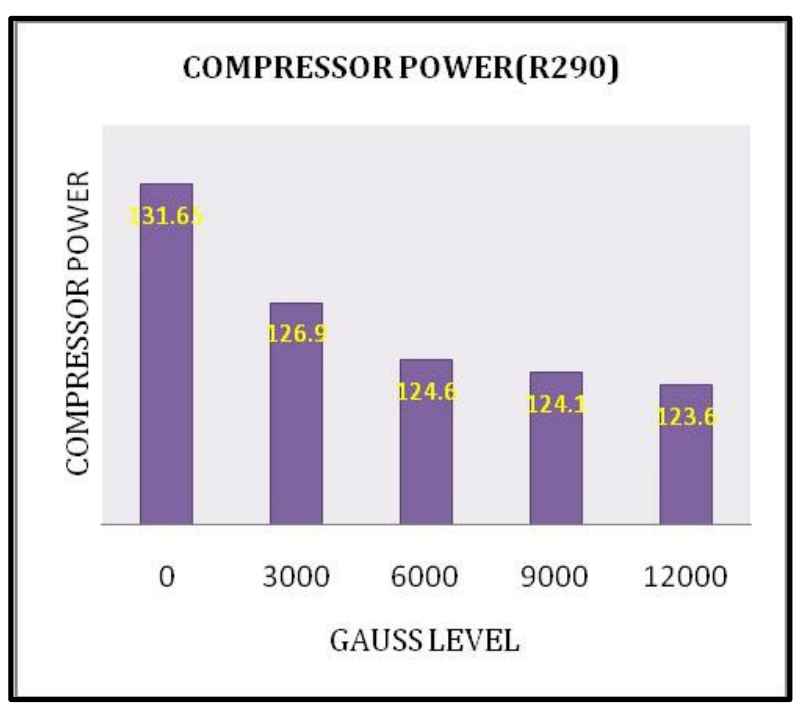

Fig. 5.Compressor Power (R290)

The compressor energy versus magnetic force of R290 is shown in Figure5. The rate of gauss increases the compressor power. The average compressor power of Gauss level 3000 is decreased by $3.60 \%$ compared to no magnetic field. The average compressor power for gauss rates 6000,9000 and 12000 is decreased by $5.35 \%$, $5.73 \%$ and $6.11 \%$ respectively.

\section{Conclusions}

During the test, the compressor energy savings of the compression cooling system was investigated under the influence of magnetic field using various refrigerants such as R134a, R151a, R407a, and R290.

The magnetic field has shown a positive effect on a decrease in vapor compression system power consumption.

The maximum power saving for R152a at 12000 gauss rate was 25.86 percent.

\section{Reference}

[1]. S.M. Sami, R.J. Kita, Behavior of new refrigerant mixtures under magnetic field, IJES, 29, 1205-1213 (2005).

[2]. A Kotb, H.E. Saad, Experimental Investigation for the Magnetic-Caloric Effect on the Refrigeration Cycle Performance, SJET, 2(3A), 339-347 (2014).

[3]. S.M. Sami, S. Aucoin, Effect of magnetic field on the performance of new refrigerant mixtures, IJES, 27, 203214 (2003).

[4]. K. Mani, V. Selladurai, Energy saving with the effect of magnetic field using R290/600a mixture as substitute

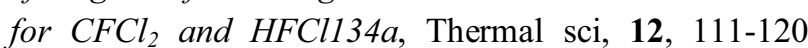
(2008).

[5]. R. Prapainop, K.O Suen (2012), Effect of refrigerant properties on refrigerant performance comparison, IJERA, 2, 486-493 (2012).

[6]. H. Guo, Z. Liu, Y. Chen, R. Yao, A study of magnetic effect on the physicochemical properties of individual hydrocarbons, Logistical Engineering College, Chongqing 400042, P.R. China(1994), 216220.

[7]. A.P. Shrotri, N.S. Mane, V.P. Patil, S.A. Wani, Magnetohydrodynamiccs emerging trend to enhance heat transfer in air conditioning system, IJSMR, 1, 41-48 (2015). 